\title{
On a Rankin-Selberg $L$-Function over Different Fields
}

\author{
Tim Gillespie \\ Department of Mathematics, St. Ambrose University, 518 W. Locust Street, Davenport, IA 52803, USA
}

Correspondence should be addressed to Tim Gillespie; gillespietimothyl@sau.edu

Received 5 February 2014; Accepted 7 April 2014; Published 27 April 2014

Academic Editor: Emrah Kılıç

Copyright (C) 2014 Tim Gillespie. This is an open access article distributed under the Creative Commons Attribution License, which permits unrestricted use, distribution, and reproduction in any medium, provided the original work is properly cited.

Given two unitary automorphic cuspidal representations $\pi$ and $\pi^{\prime}$ defined on $G L_{m}\left(\mathbb{A}_{E}\right)$ and $G L_{m}{ }^{\prime}\left(\mathbb{A}_{F}\right)$, respectively, with $E$ and $F$ being Galois extensions of $\mathbb{Q}$, we consider two generalized Rankin-Selberg $L$-functions obtained by forcefully factoring $L(s, \pi)$ and $L\left(s, \pi^{\prime}\right)$. We prove the absolute convergence of these $L$-functions for $\operatorname{Re}(s)>1$. The main difficulty in our case is that the two extension fields may be completely unrelated, so we are forced to work either "downstairs" in some intermediate extension between $E \cap F$ and $\mathbb{Q}$, or "upstairs" in some extension field containing the composite extension $E F$. We close by investigating some special cases when analytic continuation is possible and show that when the degrees of the extension fields $E$ and $F$ are relatively prime, the two different definitions give the same generating function.

\section{Introduction}

The generating function attached to pairs of automorphic representations has its origins in the papers of Rankin [1] and Selberg [2,3]. As a consequence of the knowledge of the location and multiplicities of the poles of these $L$-functions, they obtained nonvanishing results on the edge of the critical strip for Hecke's $L$-functions and asymptotic estimates for the growth of Fourier coefficients of modular forms. Moreover, considering the adelic setting in [4], Rankin-Selberg convolutions were applied to obtain more precise information regarding constant terms of Eisenstein series, and in [5] the analytic properties of Rankin-Selberg $L$-functions were used to obtain multiplicity-one results. More recently, using a version of Selberg orthogonality [6], Liu and Ye computed the $n$-level correlation function of high nontrivial zeros of automorphic $L$-functions (see [7-9]) to give insight into the distribution of prime numbers. In the classical setting as in $[4,10,11]$ we are given a Galois extension $E$ of $\mathbb{Q}$ and two automorphic cuspidal representations with unitary central characters $\pi$ on $G L_{m}\left(\mathbb{A}_{E}\right)$ and $\pi^{\prime}$ on $G L_{m^{\prime}}\left(\mathbb{A}_{E}\right)$. Suppose for any finite place $v$ of $E$ we have the associated conjugacy classes given by the Langlands correspondence

$$
\left\{\alpha_{\pi}(i, \nu)\right\}_{i=1}^{m}
$$

in $G L_{m}(\mathbb{C})$ determined by $\pi$, and

$$
\left\{\alpha_{\pi^{\prime}}(j, v)\right\}_{j=1}^{m^{\prime}},
$$

in $G L_{m^{\prime}}(\mathbb{C})$ associated with $\pi^{\prime}$. Then the finite part of the Rankin-Selberg $L$-function is given by the product of local factors

$$
L\left(s, \pi \times \pi^{\prime}\right)=\prod_{\nu<\infty} L_{\nu}\left(s, \pi \times \pi^{\prime}\right),
$$

where

$$
L_{\nu}\left(s, \pi \times \pi^{\prime}\right)=\prod_{i, j=1}^{m, m^{\prime}}\left(1-\alpha_{\pi}(i, \nu) \alpha_{\pi^{\prime}}(j, v) q_{\nu}^{-s}\right)^{-1}
$$

and $q_{\nu}$ denotes the cardinality of the residue field at a finite place $v$ of $E$. We will always write $q_{v}=p^{f_{p}}$, where $v$ lies over the prime $p$ in $\mathbb{Z}$ and $f_{p}$ denotes the modular degree (which will be the same for any $v$ lying over $p$ since $E$ is Galois) $\left[\mathcal{O}_{\nu} / \mathscr{P}_{\nu}: \mathbb{Z}_{p} / p \mathbb{Z}_{p}\right]$ where $\mathcal{O}_{\nu}$ denotes the ring of integers in $E_{v}$ with unique prime ideal $\mathscr{P}_{v}$. If a finite place $\nu$ of $E$ lies over the prime $p$ in $\mathbb{Q}$ then we will always denote $\nu \mid p$.

The Euler product in (3) is known to converge absolutely for $\operatorname{Re}(s)>1$ (see [4]), and this result is crucial in the proofs of Theorems 1 and 2. In this paper we consider two possibly 
different Galois extensions $E$ and $F$ of $\mathbb{Q}$ of degrees $\ell$ and $\ell^{\prime}$, respectively, with $\pi$ and $\pi^{\prime}$ given as before except that $\pi$ is defined on $G L_{m}\left(\mathbb{A}_{E}\right)$ and $\pi^{\prime}$ is defined on $G L_{m^{\prime}}\left(\mathbb{A}_{F}\right)$. For any prime number $p$ and any place of $E$ lying over $p$ we denote by $e_{p}$ the ramification index and $t_{p}$ the number of places of $E$ lying over the prime $p$. Thus, we have $\ell=e_{p} f_{p} t_{p}$ and we similarly denote by $e_{p}^{\prime}$ and $t_{p}^{\prime}$ the ramification index and number of places of $F$ lying over the prime $p$. So the question arises: how to define a "Rankin-Selberg" $L$-function attached to the pair $\left(\pi, \pi^{\prime}\right)$ ? We begin by forcefully factoring the standard $L$-functions of Jacquet [12]; that is, if we denote $e(x)=e^{2 \pi i x}$ for any $x \in \mathbb{C}$ then

$$
\begin{aligned}
L(s, \pi) & =\prod_{p} \prod_{\nu \mid p} \prod_{i=1}^{m}\left(1-\alpha_{\pi}(i, v) p^{-f_{p} s}\right)^{-1} \\
& =\prod_{p} \prod_{\nu \mid p} \prod_{i=1}^{m} \prod_{a=0}^{f_{p}-1}\left(1-\alpha_{\pi}(i, \nu)^{1 / f_{p}} e\left(\frac{a}{f_{p}}\right) p^{-s}\right)^{-1} ;
\end{aligned}
$$

here $v \mid p$ means the place $v$ of $E$ lies over the prime $p$ in $\mathbb{Z}$. Similarly, if $\omega$ denotes any finite place of $F$ and $f_{p}^{\prime}$ denotes the modular degree of any place of $F$ lying over the prime $p$ then

$$
\begin{aligned}
L\left(s, \pi^{\prime}\right) & =\prod_{p} \prod_{\omega \mid p} \prod_{j=1}^{m^{\prime}}\left(1-\alpha_{\pi^{\prime}}(j, \omega) p^{-f_{p}^{\prime} s}\right)^{-1} \\
& =\prod_{p} \prod_{\omega \mid p} \prod_{j=1}^{m^{\prime}} \prod_{b=0}^{f_{p}^{\prime}-1}\left(1-\alpha_{\pi^{\prime}}(j, \omega)^{1 /-f_{p}^{\prime}} e\left(\frac{b}{f_{p}^{\prime}}\right) p^{-s}\right)^{-1} .
\end{aligned}
$$

Then we define the Rankin-Selberg $L$-function as the following product:

$$
\begin{aligned}
& L\left(s, \pi \times_{E, F} \pi^{\prime}\right) \\
& =\prod_{p} \prod_{\nu, \omega \mid p} \prod_{i, j=1}^{m, m^{\prime}} \prod_{a, b=0}^{f_{p}-1, f_{p}^{\prime}-1}\left(1-\alpha_{\pi}(i, \nu)^{1 / f_{p}} \alpha_{\pi^{\prime}}(j, \omega)^{1 / f_{p}^{\prime}}\right. \\
& \left.\times e\left(\frac{a}{f_{p}}\right) e\left(\frac{b}{f_{p}^{\prime}}\right) p^{-s}\right)^{-1} .
\end{aligned}
$$

Note that Theorem 1 shows that this product is indeed well-defined. Recall from [13] that given two automorphic representations $\pi$ on $G L_{m}\left(\mathbb{A}_{E}\right)$ and $\Pi$ on $G L_{m \ell}\left(\mathbb{A}_{\mathbb{Q}}\right)$ we say that $\Pi$ is automorphically induced from $\pi$ if, for almost every prime number $p$,

$$
L_{p}(s, \Pi)=\prod_{\nu \mid p} L_{\nu}(s, \pi) .
$$

This is well-defined by the strong multiplicity-one theorem, in which case we write $A I_{E / \mathbb{Q}}(\pi)=\Pi$. So if the automorphic induction functor exists for both the extensions $E$ and $F$ and the representations $\pi$ and $\pi^{\prime}$, we could simply define

$$
L\left(s, \pi \times_{E, F} \pi^{\prime}\right)=L\left(s, A I_{E / \mathbb{Q}}(\pi) \times A I_{F / \mathbb{Q}}\left(\pi^{\prime}\right)\right),
$$

where the $L$-function on the right-hand side is the classical Rankin-Selberg $L$-function of the pair $\left(A I_{E / \mathbb{Q}}(\pi), A I_{F / \mathbb{Q}}\left(\pi^{\prime}\right)\right)$. Indeed, if $A I_{E / \mathbb{Q}}(\pi)$ and $A I_{F / \mathbb{Q}}\left(\pi^{\prime}\right)$ were known to exist globally, then $L\left(s, \pi \times_{E, F} \pi^{\prime}\right)$ would be a product of the classical Rankin-Selberg $L$-functions as given in (3).

The original motivation for considering such a convolution was to investigate the asymptotic behavior of the $n$ level correlation function (as in [14]) of high nontrivial zeros attached to a product

$$
L\left(s, \pi_{1}\right) L\left(s, \pi_{2}\right) \cdots L\left(s, \pi_{k}\right),
$$

with $\pi_{i}$ a unitary automorphic cuspidal on $G L_{m_{i}}\left(\mathbb{A}_{F_{i}}\right)$ and $F_{i}$ a Galois extension of $\mathbb{Q}$ for $i=1,2, \ldots, k$. One possible strategy is to derive a prime number theorem by investigating the asymptotic behavior of the sum as $x \rightarrow \infty$,

$$
\sum_{n \leq x} \Lambda(n) a_{\pi \times_{E, F} \pi^{\prime}}(n)
$$

where $\Lambda(n)$ denotes the Von Mangoldt function, and the coefficients $a_{\pi \times E, F} \pi^{\prime}(n)$ come from the logarithmic derivative

$$
\frac{L^{\prime}}{L}\left(s, \pi \times_{E, F} \pi^{\prime}\right)=-\sum_{n \geq 1} \frac{\Lambda(n) a_{\pi \times_{E, F} \pi^{\prime}}(n)}{n^{s}} .
$$

Once this is established one could ostensibly compute the leading order term of the $n$-level correlation function. A prime number theorem was derived in the case where $E$ and $F$ are cyclic of prime degree in [15]. Using this, the author computed the correlation function of a product $L\left(s, \pi_{1}\right) L\left(s, \pi_{2}\right) \cdots L\left(s, \pi_{k}\right)$ in [16] assuming $F_{i}$ is cyclic of prime degree for $i=1,2, \ldots, k$, thus generalizing the results of Liu and Ye [7-9]. A much more extensive goal would be to set $F=\mathbb{Q}$ and prove for a suitable collection of representations $\pi^{\prime}$ that the $L$-function $L\left(s, \pi \times_{E, F} \pi^{\prime}\right)$ enjoys "nice" analytic properties (e.g., holomorphic continuation, boundedness in vertical strips, and a functional equation); then it would follow from a converse theorem [17] that if $A I_{E / \mathbb{Q}}(\pi)$ is suitably defined as an admissible representation, then it must be automorphic.

The main problem is that beyond the case when $E$ is a cyclic extension of prime degree where the principle of functoriality holds as in [13] (or more generally a solvable extension built from towers of cyclic prime degree extensions), the properties of the global automorphic induction $A I_{E / \mathbb{Q}}(\pi)$ are not well known (see [18]). Hence, unless the principle of functoriality as in [19] is established for any number field, the analytic properties of $L\left(s, \pi \times_{E, F} \pi^{\prime}\right)$ still need to be worked out. One approach to obtain the meromorphic continuation and functional equation would be the theory of Rankin-Selberg integrals following the work of Jacquet et al. [4]. To show $L\left(s, \pi \times_{E, F} \pi^{\prime}\right)$ is bounded in vertical strips and to find the location of the poles one could try using the constant terms of Eisenstein series following the approach in [11]. Establishing the meromorphic continuation is beyond reach at this point; nonetheless, we are able to show the absolute convergence of $L\left(s, \pi \times_{E, F} \pi^{\prime}\right)$ for $\operatorname{Re}(s)>1$. We note that this result is unconditional. 
Theorem 1. Let $\pi$ and $\pi^{\prime}$ be unitary automorphic cuspidal representations of $G L_{m}\left(\mathbb{A}_{E}\right)$ and $G L_{m^{\prime}}\left(\mathbb{A}_{F}\right)$, respectively, with $E$ and $F$ being finite Galois extensions of $\mathbb{Q}$. Then $L\left(s, \pi \times_{E, F} \pi^{\prime}\right)$ converges absolutely for $\operatorname{Re}(s)>1$.

Recall that given an extension of fields $K_{1} / K_{2}$, and two automorphic representations $\pi_{K_{1}}$ and $\pi_{K_{2}}$ defined on $G L_{n}\left(\mathbb{A}_{K_{1}}\right)$ and $G L_{n}\left(\mathbb{A}_{K_{2}}\right)$, respectively, we say $\pi_{K_{1}}$ is the base change lift of $\pi_{K_{2}}$ if for any finite place $\nu_{1}$ of $K_{1}$ lying over the place $v_{2}$ of $K_{2}$ we have

$$
\left\{\alpha_{\pi_{K_{2}}}\left(j, v_{2}\right)^{f_{v_{1} \mid v_{2}}}\right\}_{j=1}^{n}=\left\{\alpha_{\pi_{K_{1}}}\left(j, v_{1}\right)\right\}_{j=1}^{n},
$$

where $\alpha_{\pi_{K_{i}}}\left(j, v_{i}\right)$ are the Satake parameters associated with $\pi_{K_{i}}$ at the place $v_{i}$ for $j=1, \ldots, n$. If the base change functor exists, we could just as well consider the convolution

$$
L\left(s, B C_{E F / E}(\pi) \times B C_{E F / F}\left(\pi^{\prime}\right)\right),
$$

where $B C_{K_{1} / K_{2}}$ denotes the base change functor for any extension $K_{1} / K_{2}$, but again this assumes functoriality which only holds for a cyclic (or more generally solvable) extension. In this vein let $\tau$ denote any finite place of the composite extension $E F$, and if $\tau$ lies over the place $v$ coming from the extension $E$, let $f_{\tau \mid \nu}$ denote the index by

$$
\left[\frac{\mathscr{O}_{\tau}}{\mathscr{P}_{\tau}}: \frac{\mathscr{O}_{\nu}}{\mathscr{P}_{\nu}}\right]
$$

where $\mathscr{O}_{\tau}$ and $\mathscr{O}_{\nu}$ denote the local rings with unique maximal ideals $\mathscr{P}_{\tau}$ and $\mathscr{P}_{\nu}$ coming from the places $\tau$ and $\nu$, respectively. Similarly, if $\tau$ also lies over the place $\omega$ coming from $F$ let $f_{\tau \mid \omega}$ denote the index by

$$
\left[\frac{\mathscr{O}_{\tau}}{\mathscr{P}_{\tau}}: \frac{\mathscr{O}_{\omega}}{\mathscr{P}_{\omega}}\right]
$$

Given $\pi$ as before, define a new set of local parameters attached to the composite extension $E F$ for any place $\tau$ of $E F$ :

$$
\left\{\alpha_{\pi_{E F}}(i, \tau)\right\}_{i=1}^{m},
$$

where if $\tau$ lies over $\nu$

$$
\left\{\alpha_{\pi_{E F}}(i, \tau)\right\}_{i=1}^{m}=\left\{\alpha_{\pi}(i, \nu)^{f_{\tau \mid \nu}}\right\}_{i=1}^{m} .
$$

Similarly, if $\tau$ lies over the place $\omega$ of $F$ define

$$
\left\{\alpha_{\pi_{E F}^{\prime}}(j, \tau)\right\}_{j=1}^{m^{\prime}}=\left\{\alpha_{\pi^{\prime}}(j, \omega)^{f_{\tau \mid \omega}}\right\}_{j=1}^{m^{\prime}} .
$$

If $q_{\tau}$ denotes the cardinality of the residue field $\mathscr{O}_{\tau} / \mathscr{P}_{\tau}$, then we define the Rankin-Selberg $L$-function

$$
\begin{aligned}
& L\left(s, \pi_{E F} \times \pi_{E F}^{\prime}\right) \\
& =\prod_{\tau} \prod_{i, j=1}^{m, m^{\prime}}\left(1-\alpha_{\pi_{E F}}(i, \tau) \alpha_{\pi_{E F}^{\prime}}(j, \tau) q_{\tau}^{-s}\right)^{-1} ;
\end{aligned}
$$

we again obtain absolute convergence for $\operatorname{Re}(s)>1$.
Theorem 2. Let $E$ and $F$ be finite Galois extensions of $\mathbb{Q}$ of degrees $\ell$ and $\ell^{\prime}$, and let $\pi$ and $\pi^{\prime}$ be unitary automorphic cuspidal representations of $G L_{m}\left(\mathbb{A}_{E}\right)$ and $G L_{m^{\prime}}\left(\mathbb{A}_{F}\right)$, respectively; then $L\left(s, \pi_{E F} \times \pi_{E F}^{\prime}\right)$ converges absolutely for $\operatorname{Re}(s)>1$.

We close by relating $L\left(s, \pi \times_{E, F} \pi^{\prime}\right)$ and $L\left(s, \pi_{E F} \times \pi_{E F}^{\prime}\right)$ when $\left(\ell, \ell^{\prime}\right)=1$ and obtain analytic continuation when $\pi$ and $\pi^{\prime}$ are the trivial representations.

Theorem 3. Let $\pi$ and $\pi^{\prime}$ be as in Theorems 2 and 1 , and suppose that $\left(\ell, \ell^{\prime}\right)=1$; then

$$
L\left(s, \pi \times_{E, F} \pi^{\prime}\right)=L\left(s, \pi_{E F} \times \pi_{E F}^{\prime}\right) .
$$

Moreover, if $1_{E}$ and $1_{F}$ denote the trivial representations of $G L_{m}\left(\mathbb{A}_{E}\right)$ and $G L_{m^{\prime}}\left(\mathbb{A}_{F}\right)$, respectively, then

$$
L\left(s, 1_{E} \times_{E, F} 1_{F}\right)=\zeta_{E F}(s),
$$

where $\zeta_{E F}(s)$ is the Dedekind zeta function of the composite extension EF. The identity (22) also holds if we only assume that $E \cap F=\mathbb{Q}$.

\section{Convergence of the Euler Products}

Proof of Theorem 1. First we expand the product

$$
\begin{gathered}
L\left(s, \pi \times_{E, F} \pi^{\prime}\right) \\
=\prod_{p} \prod_{\nu, \omega \mid p} \prod_{i, j=1}^{m, m^{\prime}} \prod_{a, b=0}^{f_{p}-1, f_{p}^{\prime}-1}\left(1-\alpha_{\pi}(i, v)^{1 / f_{p}} \alpha_{\pi^{\prime}}(j, \omega)^{1 / f_{p}^{\prime}}\right. \\
\left.\times e\left(\frac{a}{f_{p}}\right) e\left(\frac{b}{f_{p}^{\prime}}\right) p^{-s}\right)^{-1} \\
=\prod_{p} \prod_{\nu, \omega \mid p} \prod_{i, j=1}^{m, m^{\prime} f_{p}^{\prime}-1} \prod_{b=0}\left(1-\alpha_{\pi}(i, v) \alpha_{\pi^{\prime}}(j, \omega)^{f_{p} / f_{p}^{\prime}}\right. \\
=\prod_{p} \prod_{\nu, \omega \mid p} \prod_{i, j=1}^{m, m^{\prime}}\left(1-\alpha_{\pi}(i, v)^{f_{p}^{\prime}} \alpha_{\pi^{\prime}}(j, \omega)^{f_{p}} p^{-f_{p} f_{p}^{\prime} s}\right)^{-1} .
\end{gathered}
$$

Now using the bound for the local parameters as in [7] for $v$ and $\omega$ lying over the prime $p,\left|\alpha_{\pi}(i, v)\right| \leq p^{f_{p}\left(1 / 2-1 /\left(\mathrm{em}^{2}+1\right)\right)}$ and $\left|\alpha_{\pi^{\prime}}(j, \omega)\right| \leq p^{f_{p}^{\prime}\left(1 / 2-1 /\left(\ell^{\prime} m^{\prime 2}+1\right)\right)}$, then for $\operatorname{Re}(s)=\sigma$ sufficiently large we can expand into a geometric series to get

$$
\begin{aligned}
& L\left(s, \pi \times_{E, F} \pi^{\prime}\right) \\
& \quad=\prod_{p} \prod_{\nu, \omega \mid p} \prod_{i, j=1}^{m, m^{\prime}}\left(\sum_{k=0}^{\infty} \frac{\alpha_{\pi}(i, \nu)^{k f_{p}^{\prime}} \alpha_{\pi^{\prime}}(j, \omega)^{k f_{p}}}{p^{k f_{p} f_{p}^{\prime} s}}\right) .
\end{aligned}
$$


Thus by the triangle and Cauchy-Schwarz inequalities we put

$$
\begin{aligned}
& \left|L\left(s, \pi \times_{E, F} \pi^{\prime}\right)\right| \\
& \leq \prod_{p} \prod_{\nu, \omega \mid p} \prod_{i, j=1}^{m, m^{\prime}}\left(\sum_{k=0}^{\infty} \frac{\left|\alpha_{\pi^{\prime}}(i, v)\right|^{2 k f_{p}^{\prime}}}{p^{k f_{p} f_{p}^{\prime} \sigma}}\right)^{1 / 2} \\
& \quad \times\left(\sum_{r=0}^{\infty} \frac{\left|\alpha_{\pi^{\prime}}(j, \omega)\right|^{2 r f_{p}}}{p^{r f_{p} f_{p}^{\prime} \sigma}}\right)^{1 / 2} .
\end{aligned}
$$

The first sum over $k$ may be considered as a sum over the multiples of $f_{p}^{\prime}$, and similarly the sum over $r$ may be considered as a sum over multiples of $f_{p}$. Thus we get the inequality

$$
\begin{aligned}
\prod_{p} & \prod_{\nu, \omega \mid p} \prod_{i, j=1}^{m, m^{\prime}}\left(\sum_{k=0}^{\infty} \frac{\left|\alpha_{\pi^{\prime}}(i, \nu)\right|^{2 k f_{p}^{\prime}}}{p^{k f_{p} f_{p}^{\prime} \sigma}}\right)^{1 / 2} \\
& \times\left(\sum_{r=0}^{\infty} \frac{\left|\alpha_{\pi^{\prime}}(j, \omega)\right|^{2 r f_{p}}}{p^{r f_{p} f_{p}^{\prime} \sigma}}\right)^{1 / 2} \\
\leq & \prod_{p} \prod_{\nu, \omega \mid p} \prod_{i, j=1}^{m, m^{\prime}}\left(\sum_{k=0}^{\infty} \frac{\left|\alpha_{\pi}(i, v)\right|^{2 k}}{p^{k f_{p} \sigma}}\right)^{1 / 2} \\
& \times\left(\sum_{r=0}^{\infty} \frac{\left|\alpha_{\pi^{\prime}}(j, \omega)\right|^{2 r}}{p^{r f_{p}^{\prime} \sigma}}\right)^{1 / 2} \\
= & \left(\prod_{p} \prod_{\nu \mid p}^{m}\left(1-\left|\alpha_{\pi=1}(i, v)\right|^{2} p^{-f_{p} \sigma}\right)^{-1}\right)^{1 / 2} \\
& \times\left(\prod_{p} \prod_{\omega \mid p} \prod_{j=1}^{m^{\prime}}\left(1-\left|\alpha_{\pi^{\prime}}(j, \omega)\right|^{2} p^{-f_{p}^{\prime} \sigma}\right)^{-1}\right)^{1 / 2} .
\end{aligned}
$$

The first factor above is a subproduct of the classical RankinSelberg $L$-function $L(s, \pi \times \tilde{\pi})$ and the second factor is a subproduct of $L\left(s, \pi^{\prime} \times \tilde{\pi}^{\prime}\right)$. Hence these both converge absolutely for $\sigma>1$.

Proof of Theorem 2. For $\operatorname{Re}(s)=\sigma$ sufficiently large we can expand the inner factor into a geometric series

$$
\begin{aligned}
& L\left(s, \pi_{E F} \times \pi_{E F}^{\prime}\right) \\
& \quad=\prod_{\tau} \prod_{i, j=1}^{m, m^{\prime}}\left(\sum_{k=0}^{\infty} \frac{\alpha_{\pi_{E F}}(i, v)^{k} \alpha_{\pi_{E F}^{\prime}}(j, \tau)^{k}}{q_{\tau}^{k s}}\right) .
\end{aligned}
$$

Now taking absolute values and applying Cauchy-Schwarz we get

$$
\begin{aligned}
& \left|L\left(s, \pi_{E F} \times \pi_{E F}^{\prime}\right)\right| \\
& \leq \prod_{\tau} \prod_{i, j=1}^{m, m^{\prime}}\left(\sum_{k=0}^{\infty} \frac{\left|\alpha_{\pi_{E F}}(i, \tau)\right|^{2 k}}{q_{\tau}^{k \sigma}}\right)^{1 / 2} \\
& \quad \times\left(\sum_{r=0}^{\infty} \frac{\left|\alpha_{\pi_{E F}^{\prime}}(j, \tau)\right|^{2 r}}{q_{\tau}^{r \sigma}}\right)^{1 / 2} .
\end{aligned}
$$

We can write the right-hand side of the inequality as

$$
\begin{aligned}
& \left(\prod_{\nu} \prod_{\tau \mid \nu} \prod_{i=1}^{m}\left(\sum_{k=0}^{\infty} \frac{\left|\alpha_{\pi}(i, \nu)\right|^{2 k f_{\tau \mid \nu}}}{q_{\nu}^{k f_{\tau \mid \nu} \sigma}}\right)\right)^{1 / 2} \\
& \quad \times\left(\prod_{\omega} \prod_{\tau \mid \omega} \prod_{j=1}^{m^{\prime}}\left(\sum_{r=0}^{\infty} \frac{\left|\alpha_{\pi^{\prime}}(j, \omega)\right|^{2 r f_{\tau \mid \omega}}}{q_{\omega}^{r f_{\tau \mid \omega} \sigma}}\right)\right)^{1 / 2} .
\end{aligned}
$$

Since $E F$ is also a Galois extension of $\mathbb{Q}$, we have that $E F / E$ and $E F / F$ are likewise Galois, and so the numbers $f_{\tau \mid v}$ and $f_{\tau \mid \omega}$ depend only on $v$ and $\omega$, respectively. So we may write $f_{\tau \mid v}=f_{\nu}$ and $f_{\tau \mid \omega}=f_{\omega}$. Let $t_{\nu}$ and $t_{\omega}$ denote the number of places in $E F$ lying over the places $v$ and $\omega$, respectively. Note that since

$$
[E F: \mathbb{Q}]=[E F: E][E: \mathbb{Q}] \leq[E: \mathbb{Q}][F: \mathbb{Q}]=\ell \ell^{\prime},
$$

we get $[E F: E] \leq \ell^{\prime}$ and similarly $[E F: F] \leq \ell$, and thus $t_{v} \leq \ell^{\prime}$ and $t_{\omega} \leq \ell$. So we can rewrite the above product as

$$
\begin{gathered}
\left(\prod_{\nu} \prod_{i=1}^{m}\left(\sum_{k=0}^{\infty} \frac{\left|\alpha_{\pi}(i, v)\right|^{2 k f_{v}}}{q_{\nu}^{k f_{\nu} \sigma}}\right)^{t_{\nu}}\right)^{1 / 2} \\
\quad \times\left(\prod_{\omega} \prod_{j=1}^{m^{\prime}}\left(\sum_{r=0}^{\infty} \frac{\left|\alpha_{\pi^{\prime}}(j, \omega)\right|^{2 r f_{\omega}}}{q_{\omega}^{r f_{\omega} \sigma}}\right)^{t_{\omega}}\right)^{1 / 2} \\
\leq\left(\prod_{\nu} \prod_{i=1}^{m}\left(\sum_{k=0}^{\infty} \frac{\left|\alpha_{\pi}(i, \nu)\right|^{2 k f_{v}}}{q_{\nu}^{k f_{v} \sigma}}\right)\right)^{\ell{ }^{\prime} / 2} \\
\quad \times\left(\prod_{\omega}^{m_{j}^{\prime}}\left(\sum_{j=1}^{\infty} \frac{\left|\alpha_{\pi^{\prime}}(j, \omega)\right|^{2 r f_{\omega}}}{q_{\omega}^{r f_{\omega} \sigma}}\right)\right)^{\ell / 2} .
\end{gathered}
$$


By the same argument as in Theorem 1 we get the inequality

$$
\begin{gathered}
\left(\prod_{\nu} \prod_{i=1}^{m}\left(\sum_{k=0}^{\infty} \frac{\left|\alpha_{\pi}(i, v)\right|^{2 k f_{v}}}{q_{\nu}^{k f_{\nu} \sigma}}\right)\right)^{\ell^{\prime} / 2} \\
\times\left(\prod_{\omega} \prod_{j=1}^{m^{\prime}}\left(\sum_{r=0}^{\infty} \frac{\left|\alpha_{\pi^{\prime}}(j, \omega)\right|^{2 r f_{\omega}}}{q_{\omega}^{r f_{\omega} \sigma}}\right)\right)^{\ell / 2} \\
\leq\left(\prod_{\nu} \prod_{i=1}^{m}\left(\sum_{k=0}^{\infty} \frac{\left|\alpha_{\pi}(i, v)\right|^{2 k}}{q_{\nu}^{k \sigma}}\right)\right)^{\ell^{\prime} / 2} \\
\quad \times\left(\prod_{\omega} \prod_{j=1}^{m^{\prime}}\left(\sum_{r=0}^{\infty} \frac{\left|\alpha_{\pi^{\prime}}(j, \omega)\right|^{2 r}}{q_{\omega}^{r \sigma}}\right)\right)^{\ell / 2} .
\end{gathered}
$$

This last product is a subproduct of $L(s, \pi \times \tilde{\pi})^{\ell^{\prime} / 2}$ $L\left(s, \pi^{\prime} \times \tilde{\pi}^{\prime}\right)^{\ell / 2}$ and hence is absolutely convergent for $\sigma>1$.

\section{Proof of Theorem 3}

For any two Galois extensions $E$ and $F$ of $\mathbb{Q}$ consider the Dedekind zeta functions

$$
\begin{aligned}
\zeta_{E}(s) & =\prod_{p} \prod_{v \mid p}\left(1-p^{-f_{p} s}\right)^{-1} \\
& =\prod_{p} \prod_{a=0}^{f_{p}-1}\left(1-e\left(\frac{a}{f_{p}}\right) p^{-s}\right)^{-t_{p}}, \\
\zeta_{F}(s) & =\prod_{p} \prod_{\omega \mid p}\left(1-p^{-f_{p}^{\prime} s}\right)^{-1} \\
& =\prod_{p} \prod_{b=0}^{f_{p}^{\prime}-1}\left(1-e\left(\frac{b}{f_{p}^{\prime}}\right) p^{-s}\right)^{-t_{p}^{\prime}} .
\end{aligned}
$$

Taking the convolution and expanding we get the Euler product

$$
\begin{gathered}
\prod_{p} \prod_{\nu, \omega \mid p} \prod_{a=0}^{f_{p}-1} \prod_{b=0}^{f_{p}^{\prime}-1}\left(1-e\left(\frac{a}{f_{p}}\right) e\left(\frac{b}{f_{p}^{\prime}}\right) p^{-s}\right)^{-1} \\
=\prod_{p}\left(1-p^{-f_{p} f_{p}^{\prime} s}\right)^{t_{p}-t_{p}^{\prime}} .
\end{gathered}
$$

The most tractable case is when the degrees of the extensions are relatively prime, so first assume that $\left(\ell, \ell^{\prime}\right)=1$. Then since for any prime $p$ we have the identities $\ell=e_{p} f_{p} t_{p}$ and $\ell^{\prime}=e_{p}^{\prime} f_{p}^{\prime}-t_{p}^{\prime}$ and since the degree of the composite extension $E F / \mathbb{Q}$ is $\ell \ell^{\prime}$, we get that the modular degree of any prime lying in the ring of integers of $E F / \mathbb{Q}$ over $p$ is given by $f_{p} f_{p}^{\prime}$. Moreover, the number of prime ideals in the integral closure of EF lying over $p$ is $t_{p} t_{p}^{\prime}$. Thus we get that the above Euler product is the Dedekind zeta function of the composite

$$
\zeta_{E F}(s)=\prod_{p}\left(1-p^{-f_{p} f_{p}^{\prime} s}\right)^{-t_{p} t_{p}^{\prime}} .
$$

Hence we obtain analytic continuation and a functional equation for free in this case. Going back to the notation of Theorem 2 we also have that

$$
f_{\gamma \mid p}=f_{\gamma \mid \omega} f_{p}^{\prime}=f_{\gamma \mid \nu} f_{p},
$$

and since $f_{p} \mid \ell$ and $f_{p}^{\prime} \mid \ell^{\prime}$ we get $f_{p} \mid f_{\gamma \mid \omega}$ since $\left(\ell, \ell^{\prime}\right)=1$. Finally we have that $f_{\gamma \mid \omega} \mid[E F: F]$ and $[E F: F] \mid \ell$ imply that $f_{\gamma \mid \omega} \mid f_{p}$ since $f_{\gamma \mid \nu} \mid \ell^{\prime}$, so that $f_{\gamma \mid \omega}=f_{p}$. By a similar argument we get that $f_{\gamma \mid \nu}=f_{p}^{\prime}$; thus,

$$
\begin{aligned}
L\left(s, \pi_{E F} \times \pi_{E F}^{\prime}\right) & \\
& =\prod_{p} \prod_{i, j=1}^{m, m^{\prime}}\left(1-\alpha_{\pi_{E F}}(i, \tau) \alpha_{\pi_{E F}^{\prime}}(j, \tau) p^{-f_{p} f_{p}^{\prime} s}\right)^{-t_{p} t_{p}^{\prime}} \\
& =\prod_{p} \prod_{i, j=1}^{m, m^{\prime}}\left(1-\alpha_{\pi}^{f_{\gamma \mid v}}(i, \tau) \alpha_{\pi^{\prime}}^{f_{\gamma \mid \omega}}(j, \tau) p^{-f_{p} f_{p}^{\prime} s}\right)^{-t_{p} t_{p}^{\prime}} \\
& =\prod_{p} \prod_{i, j=1}^{m, m^{\prime}}\left(1-\alpha_{\pi}^{f_{p}^{\prime}}(i, \tau) \alpha_{\pi^{\prime}}^{f_{p}}(j, \tau) p^{-f_{p} f_{p}^{\prime} s}\right)^{-t_{p} t_{p}^{\prime}} \\
& =L\left(s, \pi \times_{E, F} \pi^{\prime}\right),
\end{aligned}
$$

where the last equality follows from the expansion of $L\left(s, \pi \times_{E, F} \pi^{\prime}\right)$ as in the proof of Theorem 1. If $F=\mathbb{Q}$, this is merely a restatement that the automorphic induction and base change functors are adjoint to one another (see Proposition 2.3.1 in [20] for a nice exposition).

Returning to the case of the Dedekind zeta functions, if we relax the condition that $\left(\ell, \ell^{\prime}\right)=1$ and assume that $E \cap F=$ $\mathbb{Q}$, then the situation requires more work. Since Langlands functoriality principle as in [19] follows easily, we may work with the associated Artin $L$-function. Indeed, since $\zeta_{E}(s)$ is the Artin $L$-function attached to the trivial representation $1_{E}$ of the absolute Galois group $\Gamma_{E}$, where we set $\Gamma_{K}=\operatorname{Gal}(\overline{\mathbb{Q}} / K)$ for any number field $K$, the induced representation,

$$
\operatorname{Ind}_{\Gamma_{E}}^{\Gamma_{\mathbb{Q}}}\left(1_{E}\right)=\left\{f: \Gamma_{\mathbb{Q}} \longrightarrow \mathbb{C} \mid f(h g)=f(g) h \in \Gamma_{E} ; g \in \Gamma_{\mathbb{Q}}\right\},
$$

can be canonically identified with the group algebra $\mathbb{C}\left[\Gamma_{E / \mathbb{Q}}\right]$ of the finite group $\Gamma_{E / \mathbb{Q}}=\Gamma_{\mathbb{Q}} / \Gamma_{E}=\operatorname{Gal}(E / \mathbb{Q})$. Moreover, the factorization of $\zeta_{E}(s)$ corresponds to the decomposition of $\mathbb{C}\left[\Gamma_{E / \mathbb{Q}}\right]$ into irreducibles under the right regular representation. In particular, if we denote by $\widehat{G}$ the set of equivalence classes of irreducible representations of a finite group $G$, then by [19] we can write

$$
\zeta_{E}(s)=L\left(s, \operatorname{Ind}_{\Gamma_{E}}^{\Gamma_{\mathbb{Q}}}\left(1_{E}\right)\right)=\prod_{\sigma \in \widehat{\Gamma}_{E / \mathbb{Q}}} L(s, \sigma),
$$


where $L(s, \sigma)$ denotes the Artin $L$-function attached to the irreducible representation $\sigma \in \widehat{\Gamma}_{E / \mathbb{Q}}$. By abuse of notation, we write $\sigma \in \widehat{\Gamma}_{E / \mathbb{Q}}$ to mean that we are given an irreducible representation $\bar{\sigma}$ of the finite group $\Gamma_{E / \mathbb{Q}}$ and we obtain $\sigma$ by composing with the projection

$$
\begin{gathered}
p_{E}: \Gamma_{\mathbb{Q}} \longrightarrow \Gamma_{E / \mathbb{Q}} \\
g \longrightarrow g \Gamma_{E},
\end{gathered}
$$

so that $\sigma=\bar{\sigma} \circ p_{E}$. Similarly, write

$$
\zeta_{F}(s)=\prod_{\tau \in \widehat{\Gamma}_{F / \mathbb{Q}}} L(s, \tau),
$$

with $\tau=\bar{\tau} \circ p_{F}$ for some irreducible representation $\bar{\tau}$ of $\Gamma_{F / \mathbb{Q}}$. Then the convolution may be rewritten as

$$
L\left(s, 1_{E} \times_{E, F} 1_{F}\right)=\prod_{\sigma \in \widehat{\Gamma}_{E / \mathbb{Q}}} \prod_{\tau \in \widehat{\Gamma}_{F / \mathbb{Q}}} L(s, \sigma \otimes \tau) .
$$

Note that since $E \cap F=\mathbb{Q}$ we have an isomorphism

$$
\psi: \Gamma_{E F / \mathbb{Q}} \longrightarrow \Gamma_{E / \mathbb{Q}} \times \Gamma_{F / \mathbb{Q}},
$$

given by $\psi\left(g \Gamma_{E F}\right)=\left(g \Gamma_{E}, g \Gamma_{F}\right)$. The tensor product $\sigma \otimes \tau$ factors through the subgroup $\Gamma_{E} \cap \Gamma_{F}=\Gamma_{E F}$ which gives a representation $\bar{\alpha}$ of $\Gamma_{E F / \mathbb{Q}}$. Again letting $\alpha=\bar{\alpha} \circ p_{E F}$, we get the identity

$$
L(s, \sigma \otimes \tau)=L(s, \alpha)
$$

On the other hand, suppose that we are given an irreducible representation $\bar{\alpha}$ of $\Gamma_{E F / \mathbb{Q}}$ with corresponding space $V$. Then we obtain an irreducible representation $\bar{\alpha}$ 。 $\psi^{-1}$ of the direct product $\Gamma_{E / \mathbb{Q}} \times \Gamma_{F / \mathbb{Q}}$. Thus there exist irreducible representations $\left(\bar{\sigma}, V_{1}\right)$ and $\left(\bar{\tau}, V_{2}\right)$ of $\Gamma_{E / \mathbb{Q}}$ and $\Gamma_{F / \mathbb{Q}}$, respectively, and a one-to-one and onto intertwining map

$$
\phi: V \longrightarrow V_{1} \otimes V_{2},
$$

such that for any $\left(g_{1}, g_{2}\right) \in \Gamma_{E / \mathbb{Q}} \times \Gamma_{F / \mathbb{Q}}$

$$
\phi^{-1} \circ(\bar{\sigma} \otimes \bar{\tau})\left(g_{1}, g_{2}\right) \circ \phi=\left(\bar{\alpha} \circ \psi^{-1}\right)\left(g_{1}, g_{2}\right)
$$

For any finite prime $p$ we can choose an embedding $\iota_{p}: \mathbb{Q} \hookrightarrow$ $\mathbb{Q}_{p}$ which induces a homomorphism of Galois groups

$$
\iota_{p}^{*}: \Gamma_{\mathbb{Q}_{p}} \longrightarrow \Gamma_{\mathbb{Q}}
$$

where $\iota_{p}^{*}(g)=g \circ \iota_{p}$ for all $g \in \Gamma_{\mathbb{Q}_{p}}$. Moreover, we have a short exact sequence

$$
1 \longrightarrow I_{p} \longrightarrow \Gamma_{\mathbb{Q}_{p}} \longrightarrow \operatorname{Gal}\left(\overline{\mathbb{F}_{p}} / \mathbb{F}_{p}\right) \longrightarrow 1
$$

and $\operatorname{Gal}\left(\overline{\mathbb{F}}_{p} / \mathbb{F}_{p}\right)$ is topologically generated by the Frobenius automorphism $\phi_{p}: x \mapsto x^{p}$. In other words, $\operatorname{Gal}\left(\overline{\mathbb{F}}_{p} / \mathbb{F}_{p}\right)=$ $\overline{\left\langle\phi_{p}\right\rangle}$ where the closure takes place in the Krull topology.
We choose $\operatorname{Fr}_{p} \in \Gamma_{\mathbb{Q}_{p}}$ which maps to $\phi_{p}$ and the local Artin $L$-factor at $p$ is by definition

$$
L_{p}(s, \alpha)=\operatorname{det}\left(I-p^{-s} \alpha\left(\iota_{p}^{*}\left(\operatorname{Fr}_{p}\right)\right) \mid V^{I_{p}}\right)^{-1},
$$

where $V^{I_{p}}=\left\{\nu \in V \mid \alpha(g)(\nu)=\nu\right.$ for all $\left.g \in I_{p}\right\}$. Unraveling, this gives

$$
\begin{aligned}
& L_{p}(s, \alpha) \\
& =\operatorname{det}\left(I-p^{-s}\left(\bar{\alpha}\left(\iota_{p}^{*}\left(\operatorname{Fr}_{p}\right) \Gamma_{E F}\right)\right) \mid V^{I_{p}}\right)^{-1} \\
& =\operatorname{det}\left(I-p^{-s}\left(\bar{\alpha} \circ \psi^{-1}\right)\left(\iota_{p}^{*}\left(\operatorname{Fr}_{p}\right) \Gamma_{E}, \iota_{p}^{*}\left(\mathrm{Fr}_{p}\right) \Gamma_{F}\right) \mid V^{I_{p}}\right)^{-1} \\
& =\operatorname{det}\left(I-p^{-s}\left[\phi ^ { - 1 } \circ \beta \left(\iota_{p}^{*}\left(\operatorname{Fr}_{p}\right) \Gamma_{E},\right.\right.\right. \\
& \left.\left.\left.\qquad \iota_{p}^{*}\left(\operatorname{Fr}_{p}\right) \Gamma_{F}\right) \circ \phi\right] \mid V^{I_{p}}\right)^{-1},
\end{aligned}
$$

where we have written $\beta\left(g_{1}, g_{2}\right)=(\bar{\sigma} \otimes \bar{\tau})\left(g_{1}, g_{2}\right) g_{1} \in \Gamma_{E}$, $g_{2} \in \Gamma_{F}$. Note that we have the identity

$$
\begin{aligned}
& \left(\phi^{-1} \circ \beta\left(g_{1}, g_{2}\right) \circ \phi\right) \mid V^{I_{p}} \\
& \quad=\phi^{-1} \circ \beta\left(g_{1}, g_{2}\right) \mid\left(V_{1} \otimes V_{2}\right)^{I_{p}} \circ \phi,
\end{aligned}
$$

which follows since $v \in V^{I_{p}} \Leftarrow$ for all $g \in I_{p}$

$$
\begin{aligned}
\left(\phi^{-1} \circ \beta\left(g \Gamma_{E}, g \Gamma_{F}\right) \phi\right)(v) & =\left(\bar{\alpha} \circ \psi^{-1}\right)\left(g \Gamma_{E}, g \Gamma_{F}\right)(v) \\
& =\bar{\alpha}\left(g \Gamma_{E F}\right)(v)=v
\end{aligned}
$$

if and only if

$$
\beta\left(g \Gamma_{E}, g \Gamma_{F}\right)(\phi(v))=\phi(v) \quad \forall g \in I_{p},
$$

if and only if $\phi(v) \in\left(V_{1} \otimes V_{2}\right)^{I_{p}}$, which proves the claim. Thus we can write $L_{p}(s, \alpha)$ as

$$
\begin{aligned}
\operatorname{det}\left(I-p^{-s} \beta\left(\iota_{p}^{*}\left(\mathrm{Fr}_{p}\right) \Gamma_{E}, \iota_{p}^{*}\left(\mathrm{Fr}_{p}\right) \Gamma_{F}\right) \mid\left(V_{1} \otimes V_{2}\right)^{I_{p}}\right)^{-1} \\
=\operatorname{det}\left(I-p^{-s}\left[\sigma\left(\iota_{p}^{*}\left(\mathrm{Fr}_{p}\right)\right) \otimes \tau\left(\iota_{p}^{*}\left(\mathrm{Fr}_{p}\right)\right)\right] \mid\right. \\
\left.\left(V_{1} \otimes V_{2}\right)^{I_{p}}\right)^{-1},
\end{aligned}
$$

but this is precisely $L_{p}(s, \sigma \otimes \tau)$. It follows that

$$
\prod_{\sigma \in \widehat{\Gamma}_{E / \mathbb{Q}}} \prod_{\tau \in \widehat{\Gamma}_{F / \mathbb{Q}}} L(s, \sigma \otimes \tau)=\prod_{\alpha \in \widehat{\Gamma}_{E F / \mathbb{Q}}} L(s, \alpha)=\zeta_{E F}(s) .
$$

So we again obtain all the analytic properties for free.

\section{Conflict of Interests}

The author declares that there is no conflict of interests regarding the publication of this paper. 


\section{References}

[1] R. Rankin, "Contributions to the theory of Ramanujan's function $\tau(n)$ and similar arithmetical functions, I and II," Proceedings of the Cambridge Philosophical Society, vol. 35, pp. 351-372, 1939.

[2] A. Selberg, "Bermerkungen uber eine Dirichletsche Reihe, die mit der Modulformen nahe verbunden ist," Archiv for Mathematik og Naturvidenskab, vol. 43, pp. 47-50, 1940.

[3] A. Selberg, "On the estimation of Fourier coefficients of modular forms," in Proceedings of Symposia in Pure Mathematics, vol. 8, pp. 1-15, American Mathematical Society, 1965.

[4] H. Jacquet, I. I. Piatetski-Shapiro, and J. Shalika, "RankinSelberg convolutions," The American Journal of Mathematics, vol. 105, pp. 367-464, 1983.

[5] H. Jacquet and J. A. Shalika, "On Euler products and the classification of automorphic representations I, II," The American Journal of Mathematics, vol. 103, pp. 499-558, 777-815, 1981.

[6] J. Liu and Y. Ye, "Selberg's orthogonality conjecture for automorphic L-functions," The American Journal of Mathematics, vol. 127, no. 4, pp. 837-849, 2005.

[7] J. Liu and Y. Ye, "Zeros of automorphic L-functions and noncyclic base change," in Number Theory: Tradition and Modernization, pp. 119-152, Springer, New York, NY, USA, 2005.

[8] J. Liu and Y. Ye, "Correlation of zeros of automorphic Lfunctions," Science in China, Series A: Mathematics, vol. 51, no. 7, pp. 1147-1166, 2008.

[9] J. Liu and Y. Ye, "Functoriality of automorphic $L$-functions through their zeros," Science in China, Series A: Mathematics, vol. 52, no. 1, pp. 1-16, 2009.

[10] F. Shahidi, "On certain L-functions," The American Journal of Mathematics, vol. 103, pp. 297-355, 1981.

[11] C. Moeglin and J. L. Waldspurger, "Le spectre résiduel de $G L_{n}$ ", Annales Scientifiques de l'École Normale Supérieure, vol. 22, no. 4, pp. 605-674, 1989.

[12] H. Jacquet, "Principal $L$-functions of the linear group," Proceedings of Symposia in Pure Mathematics, vol. 33, part 2, pp. 63-86, 1979.

[13] J. Arthur L, Clozel, Simple Algebras, Base Change, and the Advanced Theory of the Trace Formula, vol. 120 of Annals of Mathematics Studies, Princeton University Press, 1989.

[14] Z. Rudnick and P. Sarnak, "Zeros of principal $L$-functions and random matrix theory," Duke Mathematical Journal, vol. 88, pp. 269-322, 1996.

[15] T. Gillespie and G. Ji, "Prime number theorems for RankinSelberg L-functions over number fields," Science China Mathematics, vol. 54, no. 1, pp. 35-46, 2011.

[16] T. Gillespie, "Factorization of automorphic $L$-functions and their zero statistics," International Journal of Number Theory, vol. 9, no. 6, p. 1367, 2013.

[17] J. W. Cogdell and I. I. Piatetski-Shapiro, "Converse theorems for $G L_{n}$," Publications Mathématiques de L'Institut des Hautes Scientifiques, vol. 79, no. 1, pp. 157-214, 1994.

[18] H. H. Kim, "An example of non-normal quintic automorphic induction and modularity of symmetric powers of cusp forms of icosahedral type," Inventiones Mathematicae, vol. 156, no. 3, pp. 495-502, 2004.

[19] J. Rogawski, "Functoriality and the artin conjecture," Proceedings of Symposia in Pure Mathematics, vol. 61, pp. 331-353, 1997.

[20] D. Ramakrishnan, "Modularity of the Rankin-Selberg L-series, and multiplicity one for SL(2)," Annals of Mathematics, vol. 152, no. 1, pp. 45-111, 2000. 


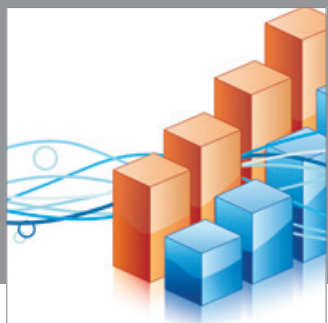

Advances in

Operations Research

mansans

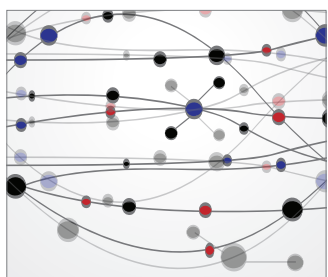

The Scientific World Journal
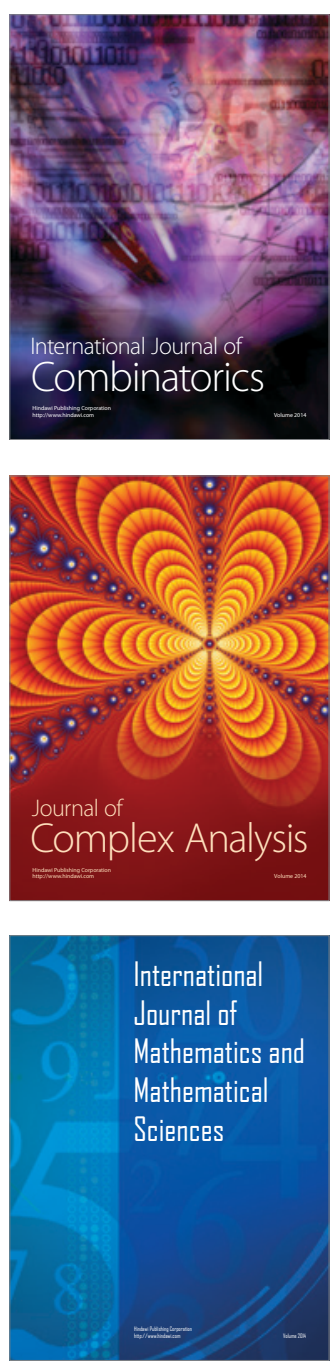
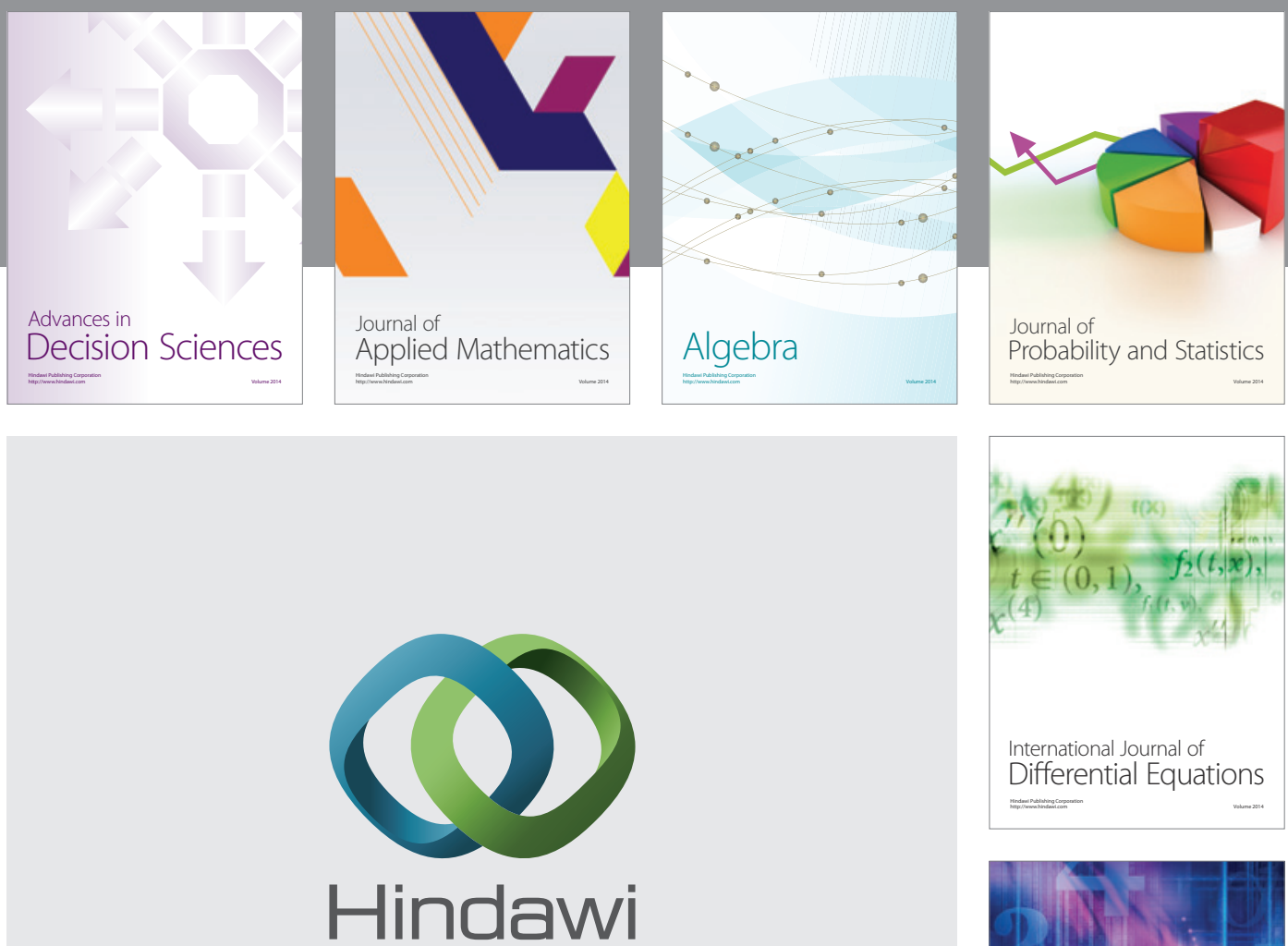

Submit your manuscripts at http://www.hindawi.com
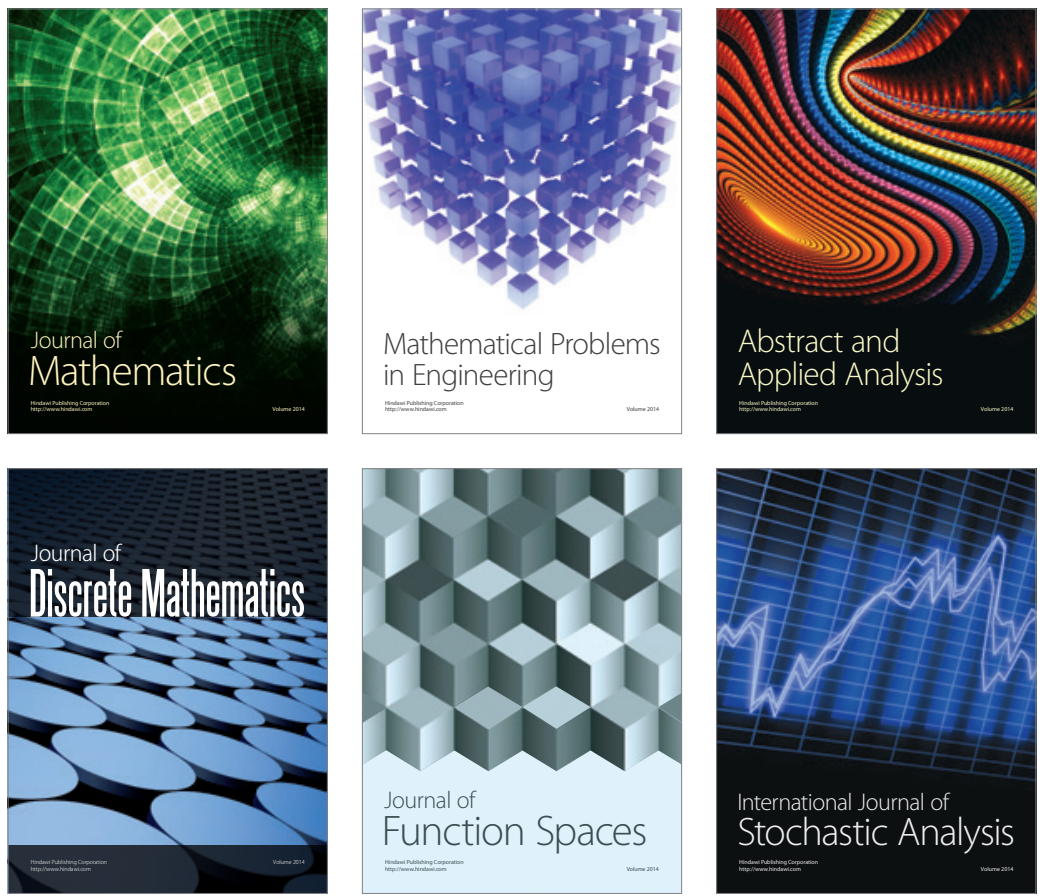

Journal of

Function Spaces

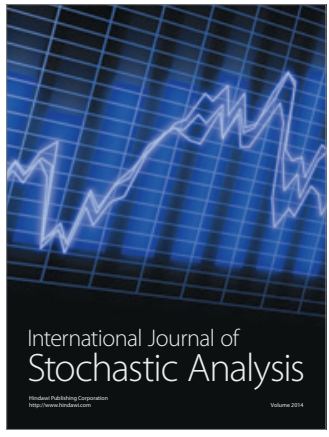

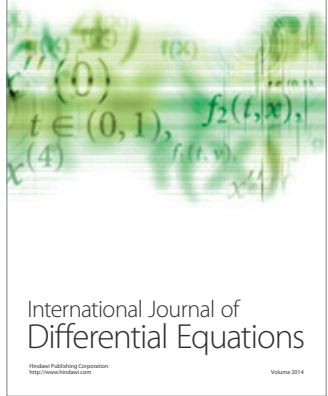
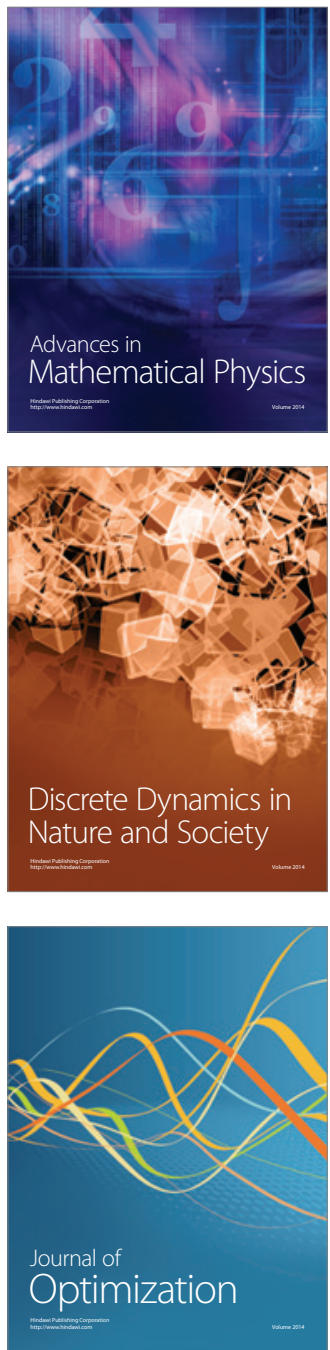